\title{
A RUNTIME STABILITY ANALYSIS OF CLOCK SYNCHRONIZATION PRECISION ON A TIME-TRIGGERED BUS PROTOTYPE
}

\author{
Fabiano C. Carvalho* \\ fabiano@mectron.com.br
}

\author{
Carlos E. Pereira ${ }^{\dagger}$ \\ cpereira@ece.ufrgs.br
}
* Mectron Engenharia, Indústria e Comércio S.A.
Av. Brigadeiro Faria Lima, 1399
São José dos Campos - Brazil
contact: fabiano@mectron.com.br
$\dagger$ Department of Electrical Engineering
Federal University of Rio Grande do Sul
Porto Alegre - Brazil
contact: cpereira@ece.ufrgs.br

\begin{abstract}
This paper provides a runtime stability analysis of the DaisyChain clock synchronization algorithm running over CASCA - a time-triggered extension of CAN bus. The main objective is to show with practical results how to achieve global time base of high precision and how this precision is affected by the modification of the TDMA transmission schedule. That contributes by providing some basic guidelines for the task of designing time-triggered, TDMA-based distributed systems for embedded control applications.
\end{abstract}

KEYWORDS: Time-Triggered Systems, Clock Synchronization, Rapid Prototyping

\section{RESUMO}

Este artigo apresenta uma análise de estabilidade do algoritmo de sincronização de relógios Daisy-Chain sendo executado sobre a plataforma CASA - um sistema de comunicação baseado no CAN bus. O objetivo é mostrar como estabelecer e manter uma base de tempo global de alta precisão. Mais ainda, o trabalho mostra a partir de resultados práticos como

Artigo submetido em 31/01/2007 (Id:778)

Revisado em 10/09/2008 e em 01/12/2008

Aceito sob recomendação do Editor Associado Prof. José Reinaldo Silva esta precisão é afetada pela modificação do padrão de comunicação TDMA. Pretende-se que este trabalho possa contribuir para o projeto de sistemas distribuidos baseados em uma arquitetura de comunicação do tipo time-triggered.

PALAVRAS-CHAVE: Sistemas Time-Triggered, Sincronização de Relógios, Prototipação Rápida

\section{INTRODUCTION}

Clock synchronization is a fundamental primitive function of time-triggered TDMA buses. Hereby, a good understanding of its operation behavior is of utmost importance for the design of derived fault-tolerant services that can be built over it. From the application level viewpoint, the quality of this service directly affects the performance of distributed control functions. For instance, the global time precision represents the varying part of message transmission delays which contributes to control jitter. Moreover, degraded precision leads to coarser grained global time granularity which results in bus bandwidth waste, since TDMA slots must be enlarged in order to account for worst case conditions and prevent from bus contention. Finally, precision degradation can also affect time-critical safety properties related to system deadlines, causality, and so on. 
Time-triggered buses are becoming a cost-effective solution for the design of safety-critical distributed systems. Examples are the FlexRay protocol, the TTCAN, the TimeTriggered Protocol and the DACAPO platform. Detailed information about each bus can be found respectively in (FlexRay, 2004), (Hartwich et al., 2002), (TTP/C, 2003), and (Rostamzadeh et al., 1995). All these buses perform essentially distributed clock synchronization by applying different mechanisms. Nevertheless, performance characteristics of these platforms are usually provided in terms of transmission speed and bus arbitration latencies but either none or very poor information is given about the stability of the clock synchronization service. In (FlexRay, 2004), for instance, it is suggested to consider a worst case precision of $11.7 \mu \mathrm{s}$ for a FlexRay bus which is calculated by using empirical equations. On the other hand, the TTP/C document claims a clock precision of $1 \mu \mathrm{s}$ but only for very stable and adequate operating conditions. An interesting theoretics stability analysis of the TTP/C clock synchronization mechanism is presented in (Nemeth, 2003). In this paper the authors provide mathematical models to help improving global time precision but physical effects like oscillators drift and message transmission delays are not considered. The TTCAN has a centralized time reference so it is relatively simple to derive clock stability analysis as shown in (Hartwich et al., 2002). Unfortunately, TTCAN suffers from lack of reliability as global time relies on a single master node which may be replicated. Replication of the TTCAN master introduces complexity and moves part of the problem to the application level. For the DACAPO platform no information on clock synchronization stability was found.

In systems with essentially distributed time base the quality of clock synchronization primitive depends on many factors, most of them related to physical characteristics like crystal oscillator drifts, bus latencies, and so on. The impact of these physical factors is difficult to predict so an experimental analysis becomes important. Another issue comes from design choices. Even though it may not be evident, it is relatively simple to show that the definition of the TDMA schedule also affects the behavior of the clock synchronization service. In this paper we investigate the impact on global time precision resulting from the modification of the TDMA transmission pattern of a distributed system prototype. The CASCA platform - a time-triggered extension of the native CAN bus was used as the underlying communication architecture.

This paper is structured as follows. Section 2 reviews main concepts of clock synchronization theory applied to TDMA systems. The CASCA platform is briefly described in section 3 . The runtime stability analysis is presented in section 4 . Section 5 closes this paper with some concluding remarks.

\section{CLOCK SYNCHRONIZATION}

The theory of clock synchronization in distributed systems is vaste and has been exhausted in the literature over the last years. For a more deep discussion in this subject the reader is referred to (Lamport and Melliar-Smith, 1985), (Srikanth and Toueg, 1987), (Ramanathan et al., 1990) and (Schneider, 1986). In this section, only the fundamental principles applicable to TDMA-based systems are reviewed and basic notation is provided.

Roughly speaking, clock synchronization is a distributed function whose objective is to guarantee that the difference between local time bases of distinct nodes do never exceed an upper limit. This upper limit is called the precision of the synchronized system, denoted by $\delta_{\max }$, and it is commonly defined by the following formula:

$$
\delta_{\max } \geq\left|C_{p}(t)-C_{q}(t)\right| \forall t
$$

in which $C_{p}(t)$ and $C_{q}(t)$ are clock readings of any two nodes $p$ and $q$, and $t$ refers to physical time.

The precision establishes a theoretical limitation on the granularity of the global time base. In practice, this limitation is never attained due to certain non-deterministic aspects related to the clock reading mechanism that are also addressed in this paper.

Another relevant definition is accuracy. It is used to provide a quantitative representation of how close the logical time base (local or distributed) is to an absolute time reference. Accuracy can be defined as:

$$
\gamma \geq|C(t)-t| \forall t
$$

in which $C(t)$ refers to system clock and $t$ refers to an absolute time reference. Clock accuracy is a secondary requirements since it is not essential to maintain synchronization over a single communication bus. This issue is not addressed in this work.

To synchronize clocks, from time to time every node collects clock readings from other nodes through the communication channel. With the set of the most recent readings a new time reference is calculated and then the local clock is corrected according to it. The time distance between two successive corrections is called the resynchronization interval and is denoted by $R$. During this interval, clock differs apart due to the effect of sublte differences with respect to nominal frequency of oscillator devices. For low cost crystal oscillators the tolerance over the nominal frequency, denoted by $\rho$, can vary from 1 to 100 parts per million. At any moment a clock 
reading is performed the impact of $\rho$ is proportional to $2 \rho R$.

Considering that, at runtime, critical control applications may use the global time feature then the clock synchronization primitive shall be at least as reliable as the function it supports. In view of that, agreement algorithms are used to minimize the effects of potential erroneous clock readings. The TTP/C bus adopts the Fault-Tolerant Average (FTA) algorithm described in (Kopetz and Ochsenreiter, 1987) while in FlexRay clock synchronization applies the Fault-Tolerant Midpoint algorithm (FTM) described in (Welch and Lynch, 1988). In both cases clock alignment is proved assuming that there is a minimal number of nonfaulty readings in the set of most recent clock samples.

On the other hand, both DACAPO and CASCA platforms use the Daisy-Chain Fault-Tolerant method presented in (Lonn, 1999). The primal difference between the Daisychain method and the previous ones is that there is no need to temporarily store clock readings during normal operation. Instead of using a set of readings to calculate a new time reference every node adjusts its local clock according to the time view of the current transmitter. At each clock reading this information is immediately used and then discarded. In the Daisy-Chain method the upper limit on the precision is formally proved in (Lonn, 1999) by assuming that the number of faulty readings per unit of time is less than a predefined rate.

The clock reading procedure implemented in TDMA buses is based on the fact that all relevant information is known a priori from the definition of the global schedule which defines what and when actions must be triggered by the system. Because of that, clock samples can be inferred rather than explicitly sent inside messages. At the moment a node perceives the frame initial pattern it takes a sample of its own clock and compare it with the time it was expecting this transmission to occur. The expecting arrival time is taken directly from the global schedule and matched against the actual arrival time resulting in what is called a deviation, or a skew, which is a good estimative of the amount of time the receiver's local clock is advanced (or late) with respect to the transmitter's.

Figure 1 shows the implicit reading mechanism implemented in CASCA. The same principle is used in FlexRay and TTP/C. At the sender side when local clock $C_{p}$ reaches $T_{0}$ an interrupt triggers the transmission of a message. The network adapter takes $\Delta_{T X}$ time units to handle this interrupt and then the first bit of the message is sent through the physical channel. The electrical signal takes $\Delta_{b u s}$ time units to propagate from the sender to the receiver. When the signal arrives at destination, another interrupt triggers a routine that takes a timestamp of the receiver local clock. From the mo-

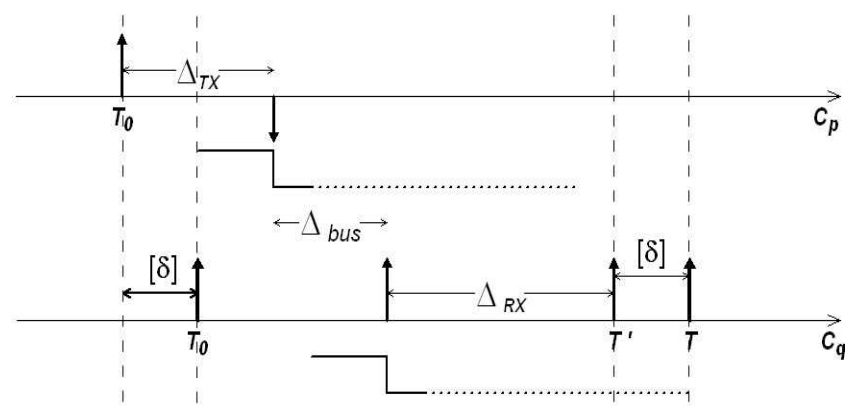

Figure 1: Clock Reading Mechanism

ment this interrupt rises to the moment the timestamp is actually taken $\Delta_{R X}$ time units have been elapsed during interrupt handling routine execution.

Let $C_{q}=T^{\prime}$ be the timestamp taken at the receiver side, and $[\delta]^{1}$ the skew between local clocks of a sender-receiver pair. The clock synchronization mechanism is interested in the value of $[\delta]$ which can be either positive or negative. Because $[\delta]$ is not explicitly available for using it may be inferred from $C_{q}$. From figure 1 we derive the equations used in the indirect reading mechanism.

First of all, we have that:

$$
T^{\prime}=T_{0}+\Delta_{T X}+\Delta_{R X}+\Delta_{b u s}+[\delta]
$$

The value of $T_{0}$, called the sender action time, is know from the TDMA schedule so, if a good estimation of network delays is available then it is possible to infer the clock skew.

It is clear that the accuracy of the clock reading procedure is strongly affected by $\Delta_{b u s}, \Delta_{T X}$ and $\Delta_{R X}$. The bus delay $\Delta_{\text {bus }}$ is intrinsic to physical properties like temperature, bus length and cable material so the variation of this term is difficult to predict. On the other hand, $\Delta_{T X}$ and $\Delta_{R X}$ depends on protocol latencies. Even if it is not possible to make these parameters absolutely deterministic the fixed part can be decoupled from the varying part like follows:

$$
\Delta_{T X}+\Delta_{R X}+\Delta_{b u s}=k+\epsilon
$$

in which $\kappa$ - the fixed part - is called compensation term and $\epsilon-$ the varying part - is called the reading error. Hereby, equation 3 can be rewritten in the form:

$$
T^{\prime}=T_{0}+k+\epsilon+[\delta]
$$

\footnotetext{
${ }^{1}$ The brackets indicates that the skew in expressed in logical time.
} 
In CASCA, the value of $k$ was precisely determined by simulation at clock cycle level. It is not a function of time but a static configuration parameter derived from platform specific implementation details. The definition of this parameters makes it possible to drastically reduce the reading error by compensating deterministic delays.

We also define the expected arrival time, denoted simply by $T$, as a reference value for the timestamp which considers zero skew:

$$
T=T_{0}+k
$$

so the value of $[\delta]$ can be inferred by subtracting the actual timestamp from the the expected arrival time as follows:

$$
T-T^{\prime}=[\delta]+\epsilon
$$

Because of $\epsilon$ the difference $T-T^{\prime}$ is not the exact deviation of $p$ and $q$ clocks but a good estimation. In practice the reading error cannot be neglected but it can be restricted to very small bounds by decoupling the varying part from the fixed part. The value of $\epsilon$ is minimized by making protocol latency variances as small as possible. For that purpose it is suggested to avoid the introduction of hubs and repeaters, and any other device in the signal path that may generate unpredictable delays.

\section{PLATFORM DESCRIPTION}

A simplistic scheme of the proposed communication platform is shown in figure 2. It has been designed as an extension of the original CAN bus specified in (CAN, 1999). The CAN bus provides physical and data link layers while the time-triggered extended layer includes advanced runtime services such as clock synchronization and TDMA arbitration.

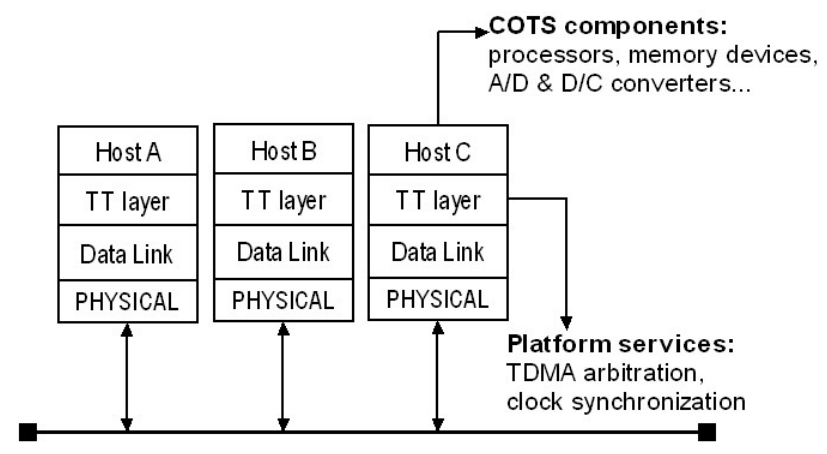

Figure 2: Proposed Communication Platform

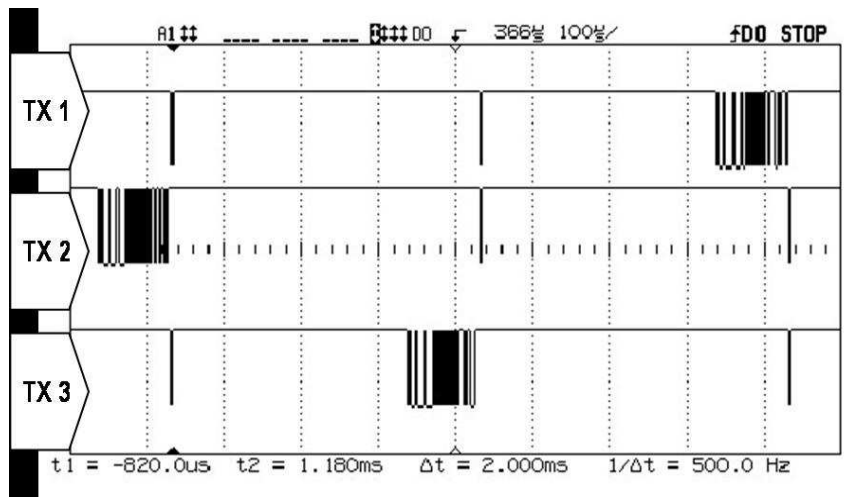

Figure 3: TDMA Arbitration

Once initialized, the network adapters operate autonomously. Messages are transmitted and received following a predefined global TDMA schedule that cannot be disturbed by timing behavior of host applications. The contents of each message - e.g. control variables and sensor samples are exchanged between the host and the network adapter through an interface memory implemented using dual-port static memories (SRAM).

The CASCA adapter core was implemented in VHDL and $\mathrm{C}$ code. It can be used free of charge for non-commercial purposes. For more detailed design information the reader is referred to (Carvalho et al., 2006a) and (Carvalho et al., 2006b).

Figure 3 shows a scope image captured during stable operation of an elementary distributed system configured with a 3 -slot, single-round TDMA network cycle. The transmission signal of each node (TX 1, 2 and 3) has been captured in order to achieve a better visualization of message exchanging.

The clock synchronization primitive implemented inside the network adapter ensure global time alignment by applying the Daisy-Chain method. Thanks to that, there is no need to rely on a specific node for the global time base to be preserved. It requires that at least two nodes remain active to ensure a bounded upper limit for $\delta_{\max }$. The principle of operation of the Daisy-Chain Algorithm is relatively simple. The network can be viewed as a logical cascade of nodes alternating the role of time leader in a repetitive sequence. The leadership order is off-line defined and coincides with the TDMA transmission order.

CASCA messages conform with standard CAN message format as shown in figure 4. Application data is allocated in the payload field for transmission. In addition, the identifier field is filled with TDMA control information that is necessary for the startup mechanism and integration of new nodes. The $r 0$ bit field is also used for safety purposes. This bit is cleared 


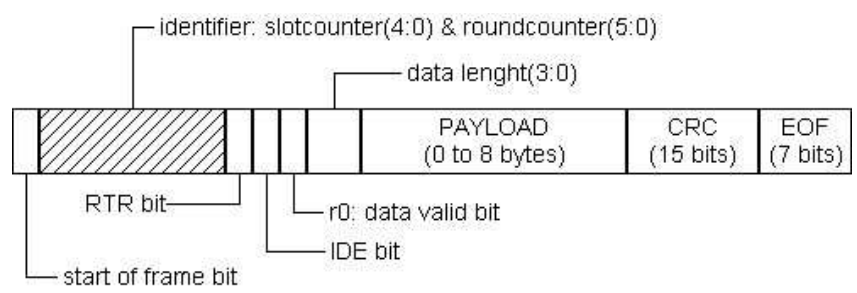

Figure 4: Message Format

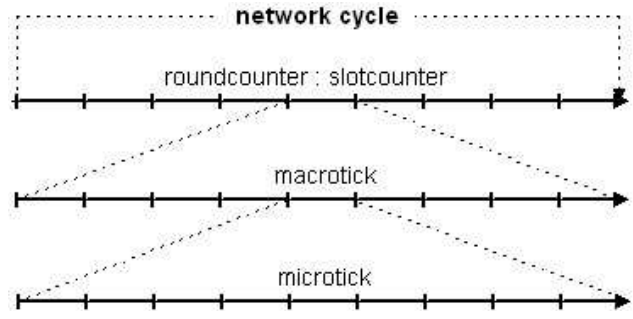

Figure 5: Time Hierarchy

whenever the payload field carries unuseful or non-updated sensor data.

Time management relies on a hierarchy of counters as indicated in figure 5. At the lowest level is microtick counter which is updated directly from the primary time source, for instance, a crystal oscillator. The size of the microtick time unit may differ from one node to another if oscillator frequencies are not equal.

At the second level is the macrotick counter which is updated whenever the microtick counter overflows. This is the only counter that is subject to corrections and its size of the macrotick must be the same for all nodes. The macrotick level defines the global time granularity.

The upper level corresponds to the system cycle counter which keeps track of the TDMA network cycle. For convenience, this counter is subdivided in round and slot counters. In CASCA, the largest network cycle that is possible to configure has 64 rounds with 32 slots each one. Being the number of slots per TDMA round a constant, the cycle counter can be expressed in the form:

[cyclecounter $]=[$ roundcounter : slotcounter $]$

By adding the minimum unit of global time - the macrotick - we have a formal representation of the global time base:

[roundcounter: slotcounter : macrotick]

This is a logical representation of the triggerable time horizon over which all communication activity of the distributed system is mapped. It makes possible to globally quantify temporal parameters like periodicity, delays, release times, and so on.

Clock synchrony in CASCA is based on phase correction, that is, the macrotick counter is suddenly modified by adding or subtracting a number of microticks. The number of microticks to be added or subtracted corresponds to the correction term, denoted by $\Lambda$, which is calculated from the most recent clock reading as follows:

$$
\Lambda=\delta \cdot 2^{-L}
$$

In which the value of $L$ depends on the size of $\delta$. Let $\mathrm{M}$ be the size of the macrotick unit so $L$ is determined according to table 1:

Table 1: [Correction term calculation criteria]

$$
\begin{array}{c|c}
0 \leq|\delta|<\frac{1}{4} \mathrm{M} & L \leftarrow 0 \\
\frac{1}{4} \mathrm{M} \leq|\delta|<\frac{1}{2} \mathrm{M} & L \leftarrow 1 \\
\frac{1}{2} \mathrm{M} \leq|\delta|<\mathrm{M} & L \leftarrow 2 \\
|\delta| \geq \mathrm{M} & L \leftarrow \infty
\end{array}
$$

The above criteria of correction term calculation prevents from large clock corrections which may lead to instability during the startup procedure. The reader is referred to (Lonn, 1999) for more information.

By using this very simple technique it is possible to maintain distributed clock alignment even if there are only two nodes communicating.

\section{RUNTIME STABILITY ANALYSIS}

The prototype setup used during runtime analysis consists in three Xilinx Spartan2E FPGA devices equipped with CAN transceiver ICs. Two nodes are connected to the extremities in order to facilitated bus termination while the third node is connected at the middle of the cable as indicated in figure 6 .

There is no application layer since host processors were not included. The platform operates autonomously sending messages with no useful information inside the payload. However, at each transmission the message identifier is filled with protocol information that is necessary for TDMA operation and startup procedure. Timing configuration parameters are summarized in table 2 .

In the central node an UART ${ }^{2}$ was synthesized inside the

\footnotetext{
${ }^{2}$ Universal Asynchronous Receiver and Transmitter
} 
Table 2: [Timing configuration parameters]

crystal frequency: $50 \mathrm{Mhz}$

microtick size: $20 \mathrm{~ns}$

macrotick size: $\quad 100$ microticks $=2 \mu \mathrm{s}$

slot size: $\quad 200$ macroticks $=400 \mu \mathrm{s}$

VHDL entity responsible for counting time. That node containing the UART is called target. This makes it possible to capture clock readings from the target into a desktop machine through a RS232 interface. Thousands of samples are collected at each prototype run in order to observe the behavior of maximum skew over time as the TDMA transmission pattern is changed.

Stability analysis is performed as shown in figure 7 . The target node always transmits in slot 0 since the beginning of each prototype run. There can be one or two stepping nodes which may change the transmitting slot at runtime in order to modify $R_{\max }$, that is, the maximum resynchronization interval. It is worthy to mention that this procedure does not affect the message transmission period. Finally, as $R_{\max }$ increases its effect is checked against the resulting worst case skew. The stability analysis procedure was divided in 2 phases: enlarged round analysis phase and reduced round analysis phase. Each phase consists in 2 prototype runs as presented next. For each prototype run results are provided by plotting over time the skew samples collected from the target node.

\subsection{Enlarged round analysis}

1. Configuration: 1 round -32 slots -2 active nodes:

System is initialized with the target node assigned to slot 0 and one stepping node assigned to slot 16 . As time progresses the stepping node jumps to slot 8 and than to slot 1 . That forced $R_{\max }$ to change from 16

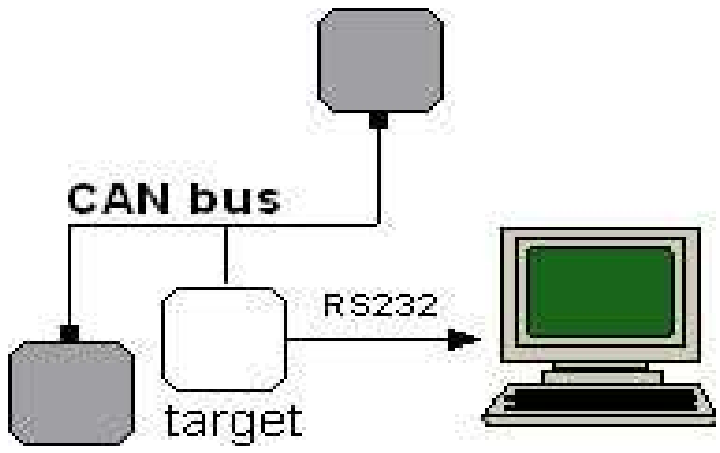

Figure 6: CASCA prototype.

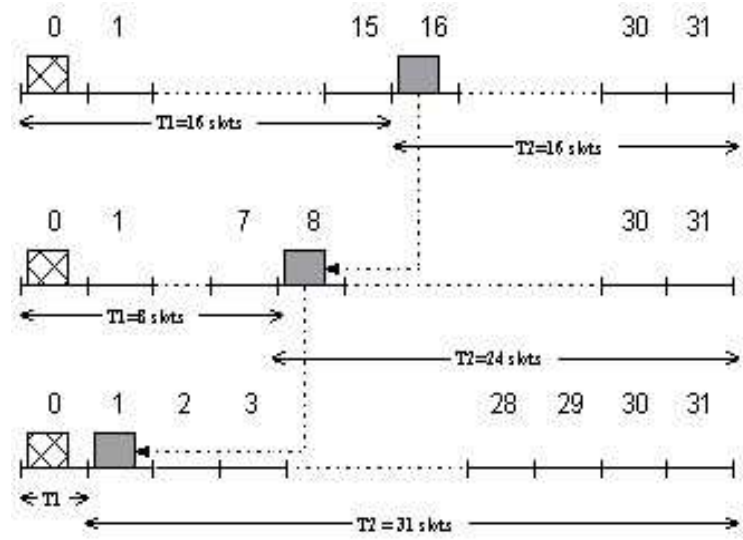

Figure 7: Node Stepping

slots $(6.4 \mathrm{~ms})$ to 24 slots $(9.6 \mathrm{~ms})$, and finally to 31 slots $(12.4 \mathrm{~ms})$. Results are shown in figure 8(a) that indicates the moment the round is reconfigured. One can observe that initially the absolute worst case skew (the distance from the zero) is near 80 microticks then it deteriorates to 90 microticks when $R_{\max }$ is increased.

2. Configuration: 1 round -32 slots -3 active nodes:

System is initialized with the target node assigned to slot 0 and two stepping nodes assigned to slots 10 and 22. As time progresses the stepping nodes jumps to slots 0 and 1 . That forced $R_{\max }$ to change from 12 slots $(4.8 \mathrm{~ms})$ to 30 slots $(12 \mathrm{~ms})$. Results are shown in figure $8(\mathrm{~b})$. The initial absolute worst case skew is 55 microticks but when $R_{\max }$ is augmented it deteriorates to 65 microticks.

\subsection{Reduced round analysis}

1. Configuration: 1 round -12 slots -3 active nodes: System is initialized with stepping nodes in slots 4 and 8 and then they jump to slots 1 and 2 . The value of $R_{\max }$ thus changes from 4 slots (1.6ms) to 10 slots ( $4 \mathrm{~ms})$. Results are shown in figure 9(a). The initial absolute worst case skew is 20 microticks $(0.4 \mu s)$ but when $R_{\max }$ is augmented it deteriorates to 30 microticks $(1.2 \mu s)$.

2. Configuration: 1 round -3 slots -3 active nodes:

In this case the stepping nodes are assigned to slots 1 and 2 up to the end of the prototype run. Results provided in figure 9 (b) represents the maximum precision attainable by this prototype.

Table 3 synthesizes the results provided in this section. The platform ensures clock alignment with only two active nodes with a worst case precision of $1.74 \mathrm{~ms}$. Furthermore, the time 


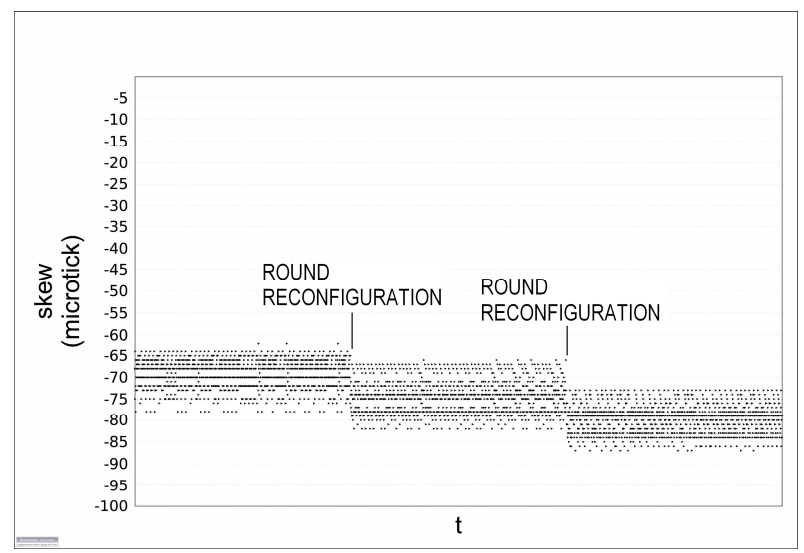

(a) Configuration: 1 round -32 slots -2 active nodes

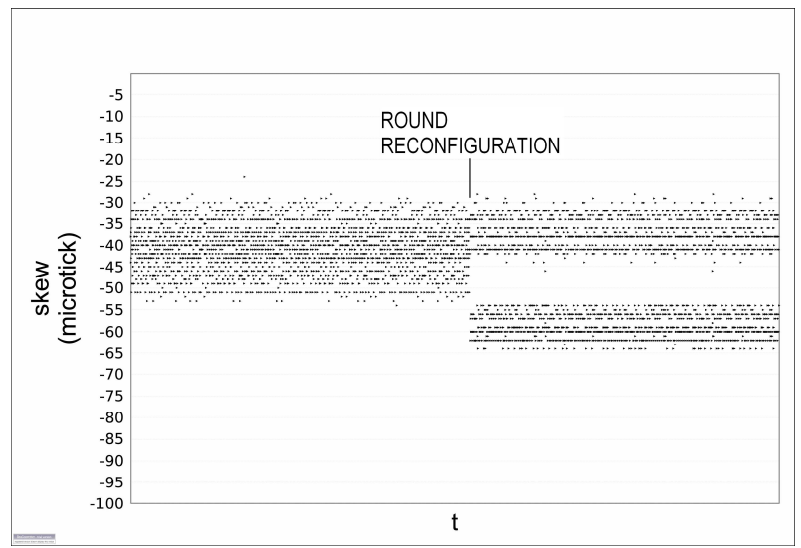

(b) Configuration: 1 round -32 slots -3 active nodes

Figure 8: Practical results - enlarged TDMA round analysis

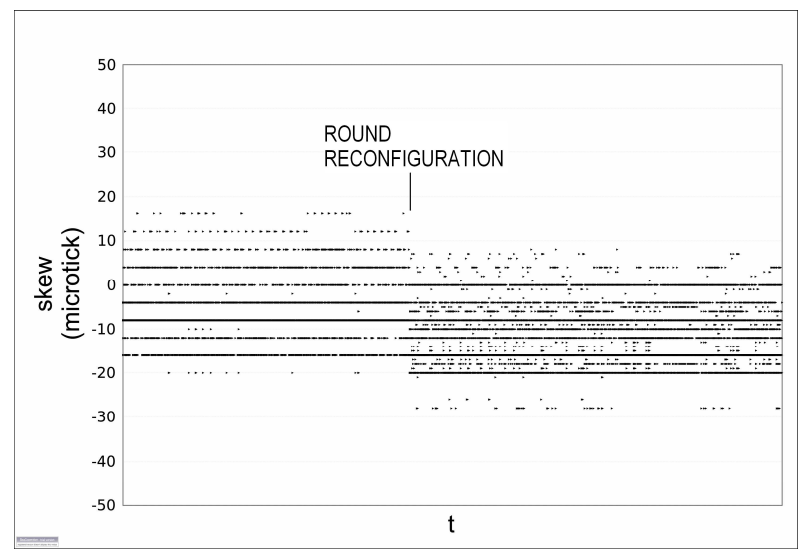

(a) Configuration: 1 round -12 slots -3 active nodes

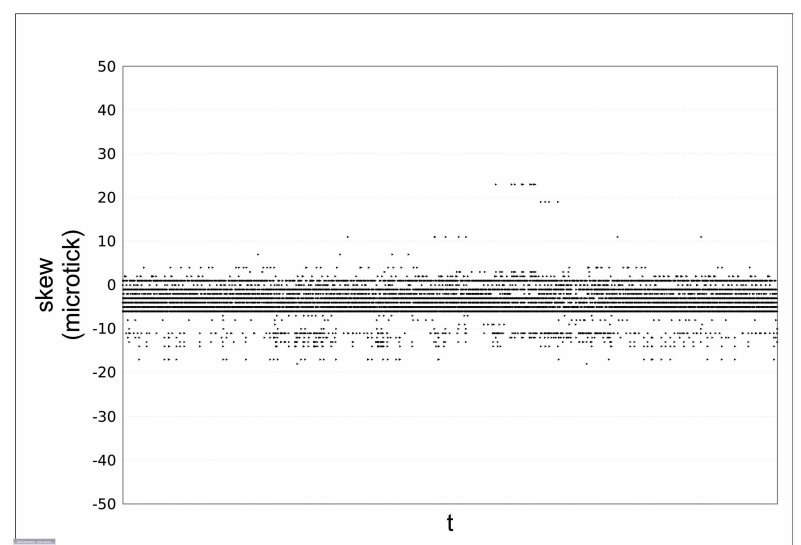

(b) Configuration: 1 round -3 slots -3 active nodes

Figure 9: Practical results - reduced TDMA round analysis

\begin{tabular}{ccccc} 
2 NODES: & & & 3 NODES: \\
\cline { 1 - 4 }$R_{\max }[m s]$ & $\delta_{\max }[\mu \mathrm{s}]$ & $R_{\max }[m s]$ & $\delta_{\max }[\mu \mathrm{s}]$ \\
6.4 & 1.56 & 0.4 & 0.36 \\
9.6 & 1.66 & 1.6 & 0.4 \\
12.4 & 1.74 & 4 & 1.2 \\
& & 4.8 & 1.1 \\
& & 12 & 1.3 \\
\hline
\end{tabular}

Table 3: [Skew analysis]

base precision is directly affected by $R_{\max }$. In the particular case when the entire channel bandwidth is used (no empty slots) the maximum resynchronization interval is minimized resulting in a global time base precision of less than $1 \mu \mathrm{s}$.

\section{FINAL REMARKS}

This paper presented a runtime stability analysis of a timetriggered communication platform. It was showed that by minimizing the maximum slot interleaving time it is possible to enhance quality of clock synchronization service without changing message transmission periods. Moreover, this paper has presented a very simple clock synchronization method to achieve a global time base precision of less than $1 \mu \mathrm{s}$ for special cases in which the bus inactivity time and hence the resynchronization interval is minimized.

\section{REFERENCES}

\section{CAN (1999). CAN Specification 2.0.}

Carvalho, F. C., Pereira, C. E., Freitas, E. P. and Silva Jr., 
E. T. (2006a). A practical implementation of the faulttolerant daisy-chain clock synchronization algorithm on can, DATE '06: Proceedings of the conference on Design, automation and test in Europe, pp. 189-194.

Carvalho, F. C., Pereira, C. E., Freitas, E. P. and Silva Jr., E. T. (2006b). A time-triggered controller area network platform with essentially distributed clock synchronization, Proceedings of the 12th IFAC Symposium on Information Control Problems in Manufacturing.

FlexRay (2004). FlexRay Communications System Protocol Specification Version 2.0, FlexRay Consortium.

Hartwich, F., Müller, B., Fuhrer, T. and Hugel, R. (2002). Timing in the ttcan network, Proceedings 8th International CAN Conference.

Kopetz, H. and Ochsenreiter, W. (1987). Clock synchronization in distributed real-time systems, IEEE Trans. Comput. 36(8): 933-940.

Lamport, L. and Melliar-Smith, P. M. (1985). Synchronizing Clocks in the Presence of Faults, Journal of the ACM 32(1): 52-78.

Lonn, H. (1999). The fault tolerant daisy chain clock synchronization algorithm, Research report, Chalmers University of Technology.

Nemeth, J. (2003). Stability analysis of distributed clock synchronization in the time-triggered architecture, Intelligent Signal Processing, 2003 IEEE International Symposium on, pp. 213-218.

Ramanathan, P., Shin, K. G. and Butler, R. W. (1990). FaultTolerant Clock Synchronization in Distributed Systems, Computer 23(10): 33-42.

Rostamzadeh, B., Lonn, H., SnedsbGl, R. and Torin, J. (1995). DACAPO - A Distributed Computer Architecture for Safety-Critical Control Applications, Proceedings of the Intelligent Vehicles Symposium, pp. 376381.

Schneider, F. B. (1986). A Paradigm for Reliable Clock Synchronization, Research report, Department of Computer Science, Cornell University.

Srikanth, T. K. and Toueg, S. (1987). Optimal clock synchronization, J. ACM 34(3): 626-645.

TTP/C (2003). Time-Triggered Protocol TTP/C High-Level Specification Document Protocol Version 1.1, TTA Group.

Welch, J. L. and Lynch, N. (1988). A new fault-tolerant algorithm for clock synchronization, Inf. Comput. 77(1): 136. 